\title{
Musculoskeletal Postoperative Infections due to Enterobacter cloacaecomplex. A New Reality?
}

Mohamed Alibashe Ahmed* and Dimitri Ceroni

Children Hospital, University Hospital Geneva, Switzerland

\begin{abstract}
From November 2011 until now, seven patients have been operated on in the Children's University Hospital of Geneva for a musculoskeletal indication and have sustained an Enterobacter cloacae complex infection. The patients presented with few clinical and laboratory manifestations. Four patients were treated with antibiotics only, whereastwo patients required debridement and vacuum assisted closure therapy associated with antibiotic therapy. One patient had his device removed.
\end{abstract}

Keywords: Subacute; External mechanisms; Osteoarticular infections; Fibrohistiocytic

\section{Introduction}

The rate of infection following orthopedic procedures has been reduced by modern techniques such as the use of peri-operative antimicrobial prophylaxis and laminar airflow in the operative theater [1]. Despite these precautions, the absolute number of infections after osteoarticular surgery is increasing because of their frequent realization; in the same manner, orthopedic implant infections are also increasing with their expanding use. Orthopedic device-related infections are classified as acute ( $<2$ weeks), subacute $(2-10$ weeks) and late $(>10$ weeks). Most of the time they are secondary to external mechanisms, and rarely due to a hematogenic pathway [2]. Most commonly cultivated bacteria in prosthetic joint infections are coagulase-negative staphylococci (30-43\%) and staphylococcus aureus (12-23\%) (1). More than $60 \%$ of these nosocomial infections are related to bacteria forming biofilms, making the diagnosis and the treatment of device-related infections difficult [3].

Enterobacter genus is a gram-negative and facultative anaerobic bacterium of the Enterobacteriaceae family. They can be found in either sewage or soils but also in the human gastrointestinal tract. Enterobacter cloacae complex (ECC) comprises six species: E. cloacae, E. hormaechei, E.asburiae, E. kobei, E. ludwigiiand E. nimipressuralis. $E C C$ are a major cause of nosocomial[bacteremia, pneumonia, urinary tract infections and postsurgical peritonitis [4]. However, ECC is an unusual cause of either orthopedic material infection or postoperative musculoskeletal lesions [5]. ECC has the ability to produce biofilm [6] and antibiotic resistance features, just asnosocomial outbreaks, are frequently correlated with these microorganisms.

No information is provided as to whether the 7 isolates were the same species or strain by any biochemical or molecular methods. In the discussion, the authors refer to these infections as an outbreak, and mention that they could not establish a common source, but reporting the relatedness of the strains would be helpful data.

To the best of our knowledge, there is currently few data in the literature concerning postoperative musculoskeletal or orthopedic device infections due to ECC. Because of the few existing hypotheses' regarding the pathogenesis, diagnosis, and management of osteoarticular infections due to ECC, we reviewed our clinical experience when dealing with this peculiar infectious process.

\section{Methods}

After approval from the Children's Hospital Review Board, medical charts of all children and adolescents who sustained postoperative osteoarticular infections at our institution were retrospectively reviewed. Based on clinical records, demographics (age and gender), body temperature, bone involved, and laboratory data, including bacterial cultures (from blood, articular fluid, or bone samples), white blood cell count (WBC), platelet count, erythrocyte sedimentation rate (ESR) and serum C-reactive protein (CRP) were collected for analysis. Radiographic studies of the affected anatomic region were available for every patient. Radiographic imaging included plain radiographs and MRI for some patients. Purulent discharge specimen, or articular fluid samples were sent to the laboratory for Gram staining and immediate inoculation onto Columbia blood agar (incubated under anaerobic conditions), CDC anaerobe 5\% sheep blood agar (incubated under anaerobic conditions), chocolate agar (incubated in $\mathrm{CO} 2$-enriched atmosphere), and brain-heart broth. The incubation time was 10 days. The protocol was approved by the local institutional review board. The study was conducted in compliance with Good Clinical Practice guidelines and the Declaration of Helsinki.

"PCR assays" not mentioned again in results or discussion to indicate what was tested by PCR and how this methodology or tests contributed to the clinical cases.

\section{Cases Presentation}

From November 2011 until now, 7 patients operated on in the Children's University Hospital of Geneva for a musculoskeletal indication sustained an ECC infection. Six patients had postoperative infections of orthopedic material. Among them, 3 cases were acute infections after percutaneous pinning of a fracture; one occurred after

*Corresponding author: Mohamed Alibashe Ahmed, Children Hospital, University Hospital Geneva, Switzerland, Tel: 02273396 23; E-mail: Mohamed.alibasheahmed@hcuge.ch

Received October 01, 2013; Accepted November 20, 2013; Published November 29,2013

Citation: Alibashe Ahmed M, Ceroni D (2013) Musculoskeletal Postoperative Infections Due to Enterobacter cloacaecomplex: A New Reality? J Med Microb Diagn 3: 128. doi:10.4172/2161-0703.1000128

Copyright: () 2013 Alibashe Ahmed M, et al. This is an open-access article distributed under the terms of the Creative Commons Attribution License, which permits unrestricted use, distribution, and reproduction in any medium, provided the original author and source are credited. 
distal osteotomy of the right tibia; one after calcaneal osteotomy, and the last after bilateral lowering of the patella. One case was not related to orthopedic device infection, but was secondary to a plexiform fibrohistiocytic tumoral lesion resection. 5 patients were boys and 2 were girls. The mean age of the patients was 9.71 years.

Most of post-operative infections (6 cases) were precocious and happened during the first 10 days following the surgery, whereas one case was detected 3 months after the operation and was thus consideredas a late infection. Clinically, ECC post-operative infections evolve usually as low-grade infection. One patient presented with fever $\left(38.5^{\circ} \mathrm{C}\right)$ and 2 patients with low-grade fever (mean $38.15^{\circ} \mathrm{C}$ ). Erythema, local pain, dehiscence of the lesion, abscess and even purulent discharge were noted in 5 patients who presented with more clinically evident signs of infection.

Blood tests were performed to assess inflammatory parameters. Four patients had normal blood leucocytes count whereas 2 had leucocytosis ( $12.1 \mathrm{G} / \mathrm{l}$ and $18.7 \mathrm{G} / \mathrm{l})$. CRP was normal in 3 patients and 3 patients had slightly increased value of their CRP (34 mg/l, $32 \mathrm{mg} / \mathrm{l}, 26$ $\mathrm{mg} / \mathrm{l})$. Three patients had thrombocytosis $(654 \mathrm{G} / \mathrm{l}, 632 \mathrm{G} / \mathrm{l}$ and $476 \mathrm{G} / \mathrm{l})$. Erythrocyte sedimentation rate was not performed routinely; it was done in 3 cases $(36 \mathrm{~mm} / \mathrm{h}, 39 \mathrm{~mm} / \mathrm{h}$ and $18 \mathrm{~mm} / \mathrm{h})$. Finally, one patient didn't have any blood tests.

Plain radiographs were performed but were not helpful for the diagnosis in any patients. MRI was performed in 2 patients and showed images compatible with infection.

All patients sustained bacteriological investigations. In 2 cases, only $E C C$ was isolated from the site of infection, whereas in the remaining 5 cases, cultures of samples demonstrated polybacterial infections. In these cases, Pseudomonas aeruginosa, Gram-positive flora, or Bacillus cereus were also found. All the ECC strains were resistant to amoxicillin and amoxicillin-clavulanate, while two strains were resistant to furanes.

E. cloacae are generally all resistant to amoxicillin and amoxicillinclavulanate, this is not unusual information. No information is provided as to whether the 7 isolates were the same species or strain by any biochemical or molecular methods.

Four patients were treated with antibiotics only, whereastwo patients required debridement and vacuum assisted closure (VAC) therapy associated with antibiotic therapy and 1 patient had his device removed. Ciprofloxacine, Cefepime or Bactrim were prescribed to the patients. The duration of antibiotic therapy ranged from 10 days with oral Bactrim to 6 weeks with oral Ciprofloxacine or iv Cefepime+oral Ciprofloxacine.

\section{Discussion}

This study constitutes, to our knowledge, the first report about musculoskeletal postoperative infections due to ECC in a paediatric population. ECC infections might even complicate minor surgeries, such as percutaneous pinning of a fracture, and affect non-debilitated children. Most of the infections are precocious and happen during the first 10 days following the surgery.

Epidemiological investigations were conducted in order to explain this outbreak of atypical postoperative infections. Sampling and bacterial analysis were made on air and surfaces of the operating facilities. Moreover, screening of the collaborators involved in the operations was also realized. However, no ECC was found, no pattern could be found with the staff and the operating rooms involved in these operations. As a consequence, the origin of the outbreak of infections could not be established.
Surprisingly, children with ECC osteoarticular infections present with few clinical manifestations although their body-temperature might occasionally be mildly elevated. Laboratory studies generally show an increased ESR. Platelet count, WBC count and CRP are typically normal or slightly increased. Radiological images are not discriminative enough, especially after a surgical procedure. Identification of the ECC on sample cultures is easy, and most postoperative musculoskeletal infections are polymicrobial.

The clinical aspects of an infection depend on the type of device, on the host characteristics and on the bacterial factors [7]. ECC has the ability to form biofilm [6], it is resistant to many antibiotics [4], and its pathogenesis is correlated to its bacterial characteristics [8].

The management of ECC musculoskeletal infections is still not standardized due to the lack of well-designed randomised prospective controlled studies [1]. However, the treatment is above all based-upon surgeon experience and infectiologists recommendations, as in the management of any prosthetic infections. Criteria for retention of the device are, signs and symptoms lasting less than 3 weeks, a diagnosis based on histopathology or microbiology, a stable implant, an absence of sinus tract and an effective available oralantibiotic [9]. Criteria were fulfilled for 4 of our patients, but only two needed surgical debridment. One patient had his device removed without replacement, and was treated with antimicrobial therapy and VAC.

For antimicrobial therapy, guidelines by the Infectious Diseases Society of America recommend the use of Cefepime $2 \mathrm{~g}$ IV q12h, Ertapenem 1g IV q24h or Ciprofloxacin 750 mg PO or 400 mg IV q12h, for 4 to 6 weeks [8].

In conclusion, ECC infections are uncommon but severe. The symptoms and signs are low-grade and the duration of treatment is long.

\section{References}

1. Zimmerli W, Trampuz A, Ochsner PE (2004) Prosthetic-joint infections. N Engl J Med 351: 1645-1654.

2. Al OB (2009) Diagnostic et traitement des infections d'implants orthopédiques Orthopédie 230: 2563-2568.

3. Murga R, Miller JM, Donlan RM (2001) Biofilm formation by gram-negative bacteria on central venous catheter connectors: effect of conditioning films in a laboratory model. J Clin Microbiol 39: 2294-2297.

4. Mezzatesta ML, Gona F, Stefani S (2012) Enterobacter cloacae complex clinical impact and emerging antibiotic resistance. Future Microbiol 7: 887-902.

5. Morand PC, Billoet A, Rottman M, Sivadon-Tardy V, Eyrolle L, et al. (2009) Specific distribution within the Enterobacter cloacae complex of strains isolated from infected orthopedic implants. J Clin Microbiol 47: 2489-2495.

6. Kim SM, Lee HW, Choi YW, Kim SH, Lee JC, et al. (2012) Involvement of curli fimbriae in the biofilm formation of Enterobacter cloacae. J Microbiol 50: $175-178$.

7. Darouiche RO (2001) Device-associated infections: a macroproblem that starts with microadherence. Clin Infect Dis 33: 1567-1572.

8. Osmon DR, Berbari EF, Berendt AR, Lew D, Zimmerli W, et al. (2013) Diagnosis and management of prosthetic joint infection: clinical practice guidelines by the infectious diseases society of america. Cli Infect Dis 56: e1-e25.

9. Widmer AF (2001) New developments in diagnosis and treatment of infection in orthopedic implants. Clin Infect Dis 33: S94-S106. 